\title{
Rehabilitación Integral en Odontología
}

\author{
Integral Rehabilitation in Dentistry
}

\section{Resumen}

La interrelación de las diversas especialidades de la odontología para la resolución de los tratamientos realizados en los pacientes que llegan a la consulta odontológica es hoy en día fundamental, ya que la visión sesgada de algún área nos puede privar el brindar una mejor posibilidad de tratamiento. Trabajar con especialistas en diferentes áreas conlleva a orientar adecuadamente los tratamientos y a optimizar resultados. En el presente artículo se detalla la rehabilitación integral de un paciente con la participación de las especialidades de cirugía oral, periodoncia, rehabilitación oral y operatoria dental. Los resultados obtenidos fueron satisfactorios para el paciente ya que se le logró devolver un esquema oclusal adecuado así como también la estética perdida. El tratamiento multidisciplinario en odontología lleva a optimizar los resultados clínicos brindando una visión más amplia de las posibilidades de tratamiento para los pacientes.

Palabras clave: rehabilitación bucal, restauración dental permanente, restauraciones de cerámica y metal

\begin{abstract}
Nowadays is fundamental the interrelationship of the diverse specialities of dentistry for the resolution of the treatments realized in the patients who come to the odontologic consultation, since the vision slanted of some area can deprive to offer a better possibility of treatment. Working with specialists in different areas carries to orientating adequately the treatments and to optimizing results. In the present article the integral rehabilitation of a patient is detailed by the participation of the specialities of oral surgery, periodoncia, oral rehabilitation and operative dental. The obtained results were satisfactory for the patient since she recover a suitably oclusal scheme as well as the lost aesthetics. The multidisciplinary treatment in dentistry leads to optimizing the clinical results offering a more wide vision of the possibilities of treatment for the patients.
\end{abstract}

Keywords: mouth rehabilitation, permanent dental restoration, metal ceramic restorations

\section{Casos Clínicos}

\section{César Lamas Lara ${ }^{1}$, Juan \\ José Paz Fernández ${ }^{2}$, Gerson \\ Paredes $\mathrm{Coz}^{2}$, Giselle Angulo \\ de la Vega ${ }^{3}$, Sully Cardoso Hernández ${ }^{4}$}

1 Cirujano Dentista, Diplomado en Odontología Restauradora y Estética de la Facultad de Odontología de la UNMSM.

2 Cirujano Dentista, Especialista en Rehabilitación Oral, Docente del Área de Rehabilitación Oral de la Facultad de Odontología de la UNMSM.

3 Cirujano Dentista, Estudiante de la Especialidad de Rehabilitación Oral de la Facultad de Odontología de la UNMSM.

4 Estudiante de internado de la Facultad de Odontología de la UNMSM.

Correspondencia:

CD César Lamas Lara

Facultad de Odontología. UNMSM.

Av. Germán Amézaga s/n. Lima 1. Perú

Teléfono: 999491403

Correo electrónico: cesar2579@hotmail.com

Fecha de recepción: 12-04-12

Fecha de aceptación: 18-07-12

\section{Introducción}

El éxito en la realización de cualquier tratamiento odontológico radica en un adecuado diagnóstico y planificación del mismo, de esta manera se logran optimizar los resultados para el beneficio de los pacientes. Es fundamental un enfoque multidisciplinario, siempre $y$ cuando el caso lo amerite, para obtener todas las perspectivas necesarias y así realizar una correcta resolución del tratamiento. La visión limitada y enfocada a una sola especialidad puede llevar a no apreciar soluciones clínicas adecuadas en la resolución de los tratamientos. ${ }^{1,2,3}$

El conocimiento de los principios estéticos, de proporción y armonía de los dientes naturales permite que el clínico pueda detectar factores que alteran la estructura de la sonrisa y de esta manera poder planificar su resolución.

Las coronas metalocerámicas han sido utilizadas con éxito desde que fueron introducidas en 1956 por Brecker. ${ }^{4} \mathrm{Y}$ sus usos en odontología han sido ampliamente descritos. A pesar de contar con algunas desventajas como son los problemas estéticos básicamente, todavía son considerados como una muy buena opción en una rehabilitación integral ya que ofrecen una alta predictibilidad. 5,6

Para realizar preparaciones en prótesis fija se debe considerar ciertos principios básicos que conllevan a optimizar resultados. Se debe tratar de ser conservadores en las preparaciones para con- servar la vitalidad de las piezas dentarias a tallar. Para realizar preparaciones en prótesis fija que tengan una buena retención se debe lograr cierto grado de conicidad. Se considera ideal lograr un grado de convergencia hacia oclusal de las paredes entre 2 a 6.5 grados. $^{7}$

El éxito de un tratamiento con prótesis fija se basa en tres criterios: vitalidad pulpar, salud gingival y longevidad de la prótesis. Aproximadamente el $50 \%$ de los dientes involucrados en una prótesis fija requieren tratamiento de conductos, ${ }^{8}$ entonces se debe hacer todo lo posible para mantener la vitalidad del otro $50 \%$.

Existen muchos conceptos sobre oclusión dentaria, y estos conceptos se encuentran a veces muy alejados de 
la práctica clínica del odontólogo9 . El incorporar estos conceptos al desarrollo de la actividad profesional conlleva a una adecuada resolución de los casos devolviendo así la función del sistema estomatognático.

Cabe destacar que el uso del articulador semiajustable en rehabilitaciones con prótesis fija es muy importante ya que este brinda la posibilidad de reproducir los movimientos mandibulares del paciente. Entre más preciso sea este articulador mejor será utilizarlo en la consulta clínica. ${ }^{10}$

El objetivo del caso fue devolver al paciente una adecuada función masticatoria y la estética perdida a través del manejo odontológico multidisciplinario.

\section{Reporte del caso}

Paciente de sexo femenino de 45 años de edad que acude a la Clínica de la Facultad de Odontología de la Universidad Nacional Mayor de San Marcos para realizarse una rehabilitación integral preocupada por su problema bucal.

Al examinar a la paciente se puede apreciar restauraciones defectuosas con amalgama dental y presencia de lesiones de caries recidivante, corona defectuosa tipo Veneer en la pieza 2.4, piezas ausentes y desgaste marcado del sector anterior. (Figura 1, 2 y 3).

Se realizó la toma de radiografías periapicales seriadas y panorámica, y su posterior estudio radiográfico. (Figura 4)

Se procedió a la toma de modelos de estudio, su posterior articulado para el correcto análisis de la oclusión y un encerado de diagnóstico. (Figura 5, 6 y 7).

\section{Diagnóstico}

Del estado de salud general: Paciente con buen estado de salud general, sin riesgo sistémico al tratamiento estomatológico.

Del estado de salud estomatológico: $\mathrm{Pa}-$ ciente con:

Caries dental: presencia de restauraciones defectuosas y caries recidivante en piezas $1.7(\mathrm{O}), 1.5(\mathrm{OD}), 2.6(\mathrm{O})$ y 4.7 (O).

Edéntula parcial: ausencia de piezas $1.8,1.6,2.2,2.4,2.7,3.8,3.6,4.6,4.8$

Pieza dentaria 2.7 vestibularizada, sin contacto oclusal.
Presencia de corona defectuosa tipo Veneer en pieza 2.4 .

Reborde alveolar reducido a nivel de la pieza 2.2

Oclusión alterada: plano oclusal alterado, alteración de la guía anterior, curva de Spee alterada.

\section{Plan de tratamiento}

Paciente sin riesgo al tratamiento estomatológico

Fisioterapia oral, enseñanza de la técnica de cepillado, hilo dental y enjuague bucal.

Restauraciones directas con resina compuesta en piezas $1.7(\mathrm{O}), 1.5(\mathrm{OD}), 2.6$ (O) y $4.7(\mathrm{O})$.

Exodoncia de la pieza 2.7 (vestibularizada)

Alargamiento de Corona Clínica del sector anterosuperior e Injerto Mixto (conjuntivo y epitelial) en el reborde alveolar a nivel de la pieza 1.2

Prótesis fijas metal cerámica en las piezas $1.4,1.3,1.2$ y 1.1 y prótesis parciales fijas metal cerámica en las piezas 1.7 y $1.5,2.1$ у $2.3,2.4$ y $2.6,3.7$ y 3.5 , 4.5 y 4.7

Confección de una férula miorelajante.

Reforzamiento de la enseñanza de la técnica de cepillado, uso del hilo dental y enjuague bucal para el mantenimiento adecuado de la rehabilitación protésica

\section{Tratamiento}

Se procedió a realizar el refuerzo de la técnica de cepillado, el uso del hilo dental y el enjuague bucal.

En una cita posterior se realizó las restauraciones con resina con resina compuesta en la piezas $1.7(\mathrm{O}), 1.5(\mathrm{OD})$, $2.6(\mathrm{O})$ y $4.7(\mathrm{O})$ y la extracción de la pieza 2.7 por encontrarse fuera de arco dentario, vestibularizada y con pobre soporte óseo para ser considerada como pilar de prótesis fija.

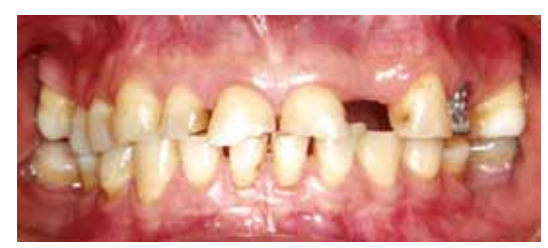

Fig. 1. Vista Inicial en Máxima Intercuspidación

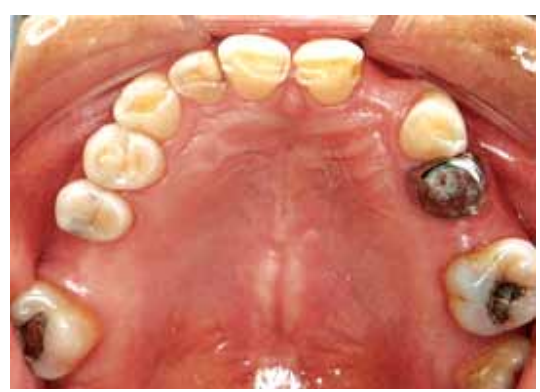

Fig. 2. Vista Inicial Oclusal Superior

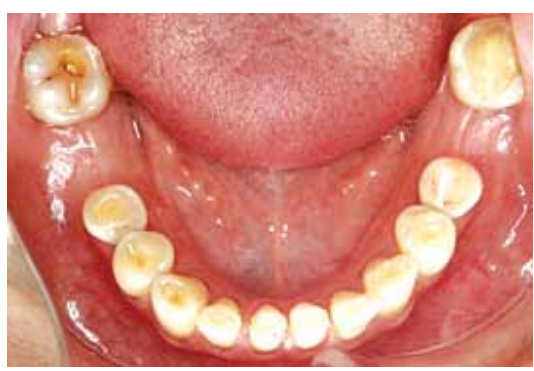

Fig. 3. Vista Inicial Oclusal Inferior

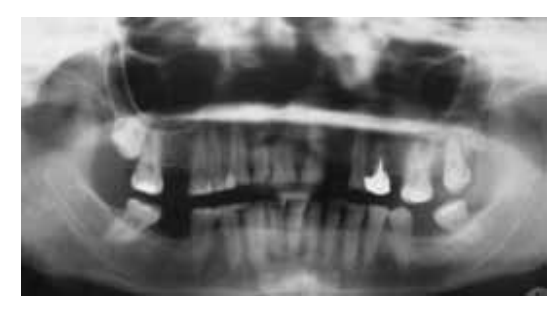

Fig. 4. Radiografía panorámica

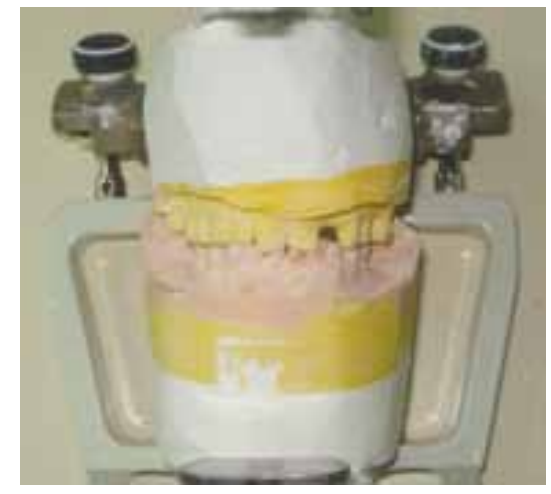

Fig. 5. Modelos de Estudio articulados en ASA.

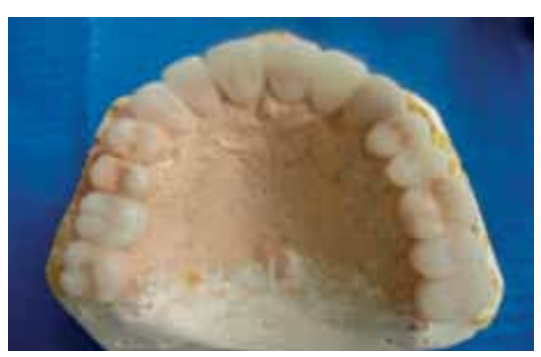

Fig. 6. Encerado de Diagnóstico, Modelo Superior

Odontol. Sanmarquina 2012; 15(1):31-34 


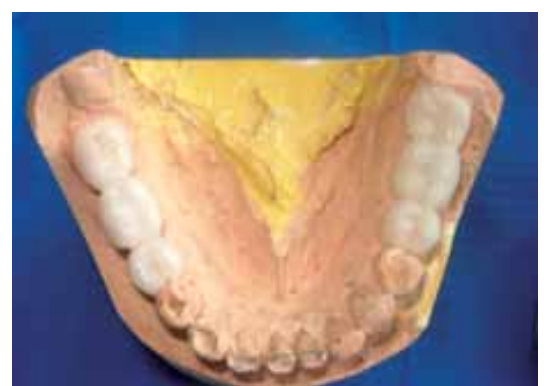

Fig. 7. Encerado de Diagnóstico, Modelo Inferior

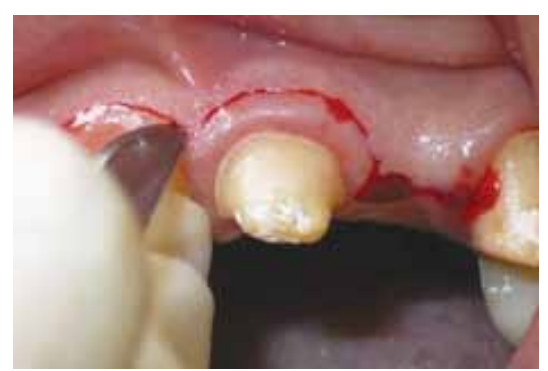

Fig. 8. Alargamiento de Corona Clínica e Injerto de Tejido Epitelial y Conectivo

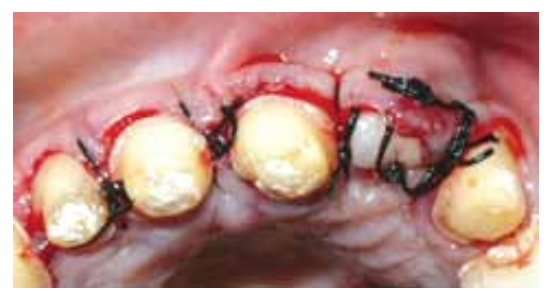

Fig. 9. Postoperatorio inmediato

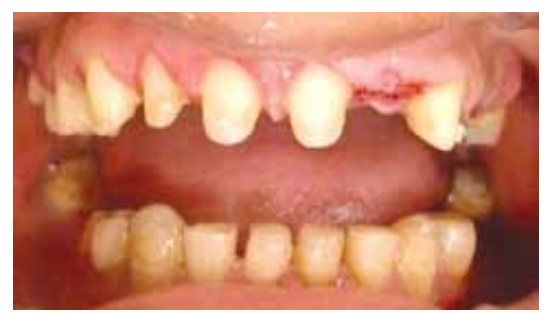

Fig. 10. Quince días después del procedimiento quirúrgico

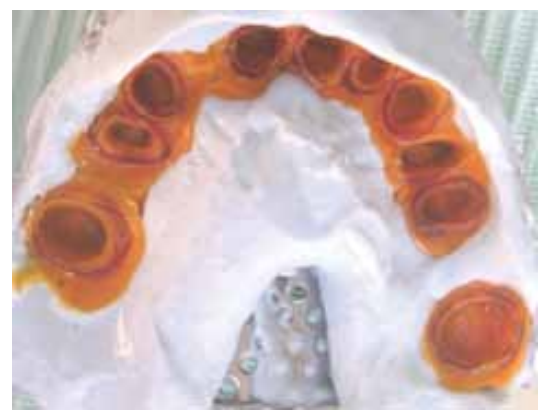

Fig. 11. Toma de Impresión Superior con Copas de Ripol

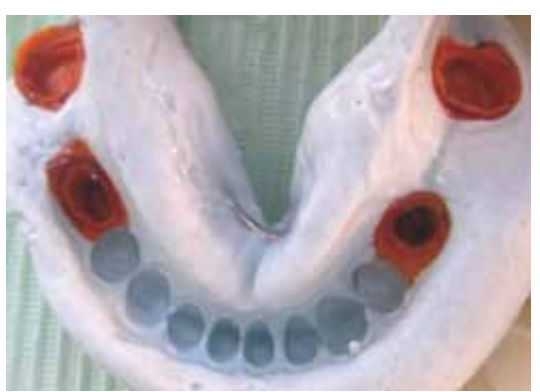

Fig. 12. Toma de Impresión Inferior con Copas de Ripol

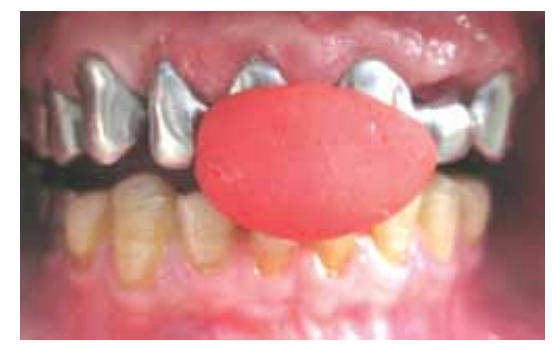

Fig. 13. Prueba de las Estructuras Metálicas

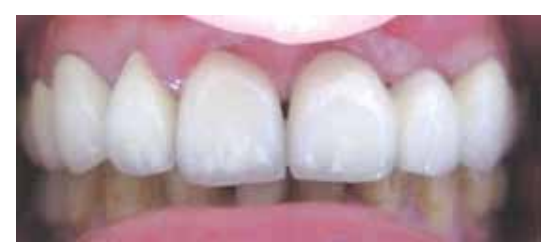

Fig. 14. Prueba de Biscocho

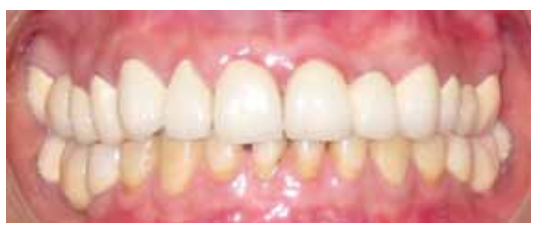

Fig. 15. Prótesis Fijas Terminadas

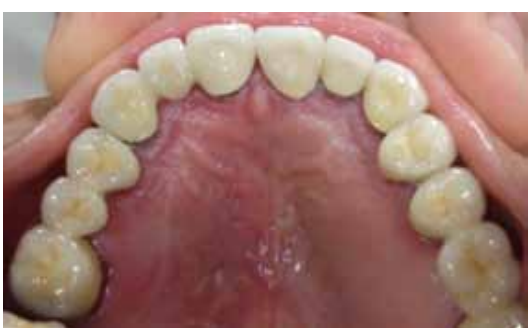

Fig. 16. Vistas Oclusal Superior

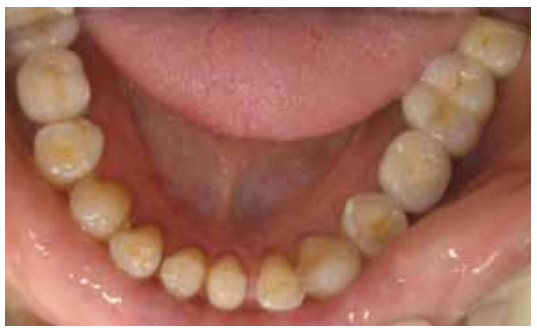

Fig. 17. Vista Oclusal Inferior

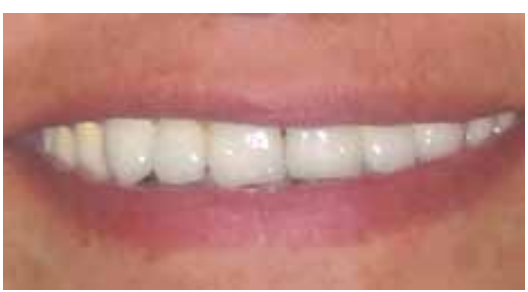

Fig. 18. Fotografía de Control 18 meses después

Se procedió a realizar las preparaciones dentarias para coronas individuales en las piezas $1.4,1.3,1.2$ y 1.1 y preparaciones para prótesis parciales fijas en las piezas 1.7 y $1.5,2.1$ y $2.3,2.4$ y $2.6,3.7$ y $3.5,4.5$ y 4.7 y los provisionales respectivos en base a una matriz de acetato y al encerado de diagnóstico.

Considerando que las piezas dentarias en el sector antero superior son piezas con una altura coronal corta; para obtener una mejor altura del muńón se realizó un alargamiento de corona clínica de ese sector (Figura 8) y considerando que el reborde alveolar a nivel de la pieza 2.2 está colapsado en sentido bucopalatino; se procedió a realizar un injerto mixto para mejorar la calidad del reborde en el mismo procedimiento quirúrgico (Figura 9 y 10).

Se esperó un tiempo prudencial de 1 mes hasta que los tejidos blandos se recuperen y se procedió al retallado y a la toma de impresión con la técnica de copas de Ripol y arrastre con alginato. (figura 11 y 12) Posteriormente se realiza el vaciado con yeso extraduro. Se tomó registro con arco facial y registros intermaxilares para poder articular los modelos de trabajo en ASA.

Una vez articulados y troquelados los modelos se envió al laboratorio dental para la confección de las estructuras metálicas.

Se realizó la prueba de estas estructuras metálicas en boca verificando que ingresen correctamente (Figura 13). Se seleccionó el color de la cerámica a aplicar siendo el color elegido como cuerpo A1 de la escala VITA.

En una cita posterior se prueban las coronas metal porcelana verificando el color de las piezas y la oclusión tanto en céntrica como en excéntrica (Figura 14). Se obtuvo la conformidad del color, oclusión y de la forma de las coronas por parte del paciente y se envían de nuevo al laboratorio para la realización del glaseado de las coronas.

En un cita posterior se realizó la cementación provisional con un cemen- 
to temporario de las coronas y puentes metalcerámica (Figura 15, 16 y 17) y se confecciona una férula miorelajante para proteger a los dientes naturales y verificar la comodidad de las prótesis fijas cuando el paciente realiza su función masticatoria y de fonación.

Posteriormente se realizó la cementación definitiva con ionómero de vidrio. Se citó a la paciente para realizarle controles periódicos y evaluar el estado de la rehabilitación (Figura 18).

\section{Resultados}

Los resultados obtenidos fueron satisfactorios para la paciente ya que se logró devolver un esquema oclusal adecuado logrando así la función del sistema estomatognático.

Desde el punto de vista estético, si bien es cierto las coronas metalocerámicas no logran los resultados ópticos que sí dan las coronas cerámicas sin metal, en este caso se logró devolver una sonrisa armoniosa y una buena naturalidad en el sector anterosuperior.

\section{Discusión}

Las restauraciones metal cerámicas presentan una excelente biocompatibilidad y una buena estética, es por ello que su uso es muy frecuente en la práctica odontológica. A pesar de la evolución mecánica de las estructuras de cerámicas, en casos extensos el uso de estructuras de metal es más recomendado al presentar mayor resistencia a la fractura encontrándose un índice de 5 a $10 \%$ en 10 años de uso. ${ }^{11,12 \text { y } 17}$

En la actualidad la cerámica sin metal está teniendo un auge importante en restauración de dientes con tratamiento de conductos y severa destrucción coronal ${ }^{18-20}$ pero en situaciones clínicas de rehabilitaciones de prótesis parcial fija extensas no hay un seguimiento que demuestre su éxito en un periodo largo de tiempo ${ }^{13,14}$ a diferencia de las restauraciones metal cerámicas que sí cuentan con estudios que avalan su uso.

Una de las ventajas de las restauraciones metal cerámicas es la posibilidad de su reparación en caso de fracturas (dependiendo del tipo de fractura que se presente) valiéndonos de técnicas directas o indirectas reduciendo el costo y tiempo. ${ }^{15,16}$

El tratamiento multidisciplinario nos lleva analizar de una mejor manera las situaciones clínicas que se presentan; haciendo participe a las diferentes especialidades para brindar mejores alternativas de tratamiento. ${ }^{3,20}$

\section{Conclusiones}

El tratamiento multidisciplinario en odontología nos lleva a optimizar resultados clínicos, brindándonos una visión más amplia de las posibilidades de tratamiento hacia los pacientes.

En la resolución de los casos es fundamental un adecuado diagnóstico de las patologías orales que pueden afectar al paciente, es por ello que el odontólogo de práctica general debe de conocer todas las áreas de la odontología y así realizar un adecuado diagnóstico, así mismo consultando con los demás especialistas realizar un adecuado plan de tratamiento para el beneficio del paciente.

Las rehabilitaciones orales con prótesis fija metal cerámicas ofrecen a los pacientes una buena posibilidad tanto funcional como estética ya que este tipo de restauraciones presentan buenas propiedades tanto mecánicas como ópticas.

\section{Referencias bibliográficas}

1. Cadafalch GE, Cadafalch CJ. Manual clínico de Prótesis fija. Madrid: Harcourt Brace; 1997. 94p.

2. Lara TL, Ochoa TJ, Gaitán VJ, Herrera CM. Rehabilitación con coronas completas de metal-cerámica y prótesis parciales removibles convencionales. Odontol. Sanmarquina $2009 ; 12(2)$ : 78-82

3. Watanabe VR, Salcedo MD, Ochoa TJ, Horna PH, Herrera CM, Paz FJ. Rehabilitación oral con prótesis fija. Odontol. Sanmarquina 2008; 11(2): 96-99

4. Brecker, C. S., Porcelain baked to gold: A new médium in prosthodontics. J. Prosthet. Dent, 1986; 23(6):801-811.

5. Mallat DE. Fundamentos de la estética bucal en el grupo anterior. Barcelona. Quintessence 2001. 55-101 p.

6. Romeo M, Martínez JA, Vallejo J. Introducción a los sistemas CAD/ CAM de uso en clínica y laboratorio. Rev. Int. Prot. Estomatol. 2005; 7(5): 524-534

7. Guyer, S. E. Multiple preparation for fixed prosthodontics. J. Prosthet. Dent. 1970; 23:529-553, 1970 .

8. Pegoraro, L. F.; Prótesis Fija. Sao Paulo, Artes Medicas, 2001. 45-47p

9. Gutierrez M y col, Importancia de la Oclusión Dentaria en la Rehabilitación por Prótesis Parcial Fija. Rev Cubana Estomatol 2001; 38(3):155-64
10. Kiliaridis S, Johansson A, Haraldson T, Omar R, Carlsson GE. Craneofacial morphology, occlussal traits, and bite force in persons with advanced occlusal tooth wear. Am J Orthod Dentofac Orthop 2000; 107(83):286-99.

11. Wasserman A, Kaiser M, Stnub JR. Clinical long-term results of VITA In-Ceram classic crowns and fixed partial dentures: A systematic literature review. Int J Prosthodont 2006;19(1):355-363.

12. Malament KA, Grossman DG. Bonded vs. nonbonded Dicor crowns: Four years report (abstract). J Dent Res 1992; 71(1) :321-323.

13. Pospiech P, Rammelsberg P, Unsold F, Gernet W. Four-year clinical evaluation of In-Ceram adhesive bridges: Preliminary results. J Dent Res 1996;75(5):147-151.

14. Suárez MJ, Lozano JF, Salido MP, Martínez F. Three-year clinical evaluation of In-Ceram Zirconio posterior FPDs. Int J Prosthodont 2004; 17(6):35-38.

15. Fraxino B, Goulart CR, Caregnatto ME, Pedroso LM, Leite MS. Técnica Indirecta Para Reparación De Prótesis Parcial Fija Metal-Porcelana Relato del Caso. Acta odontol. venez. 2010; 48(1): 164-169

16. Ozcan M. Evaluation of alternative intra-oral repair techniques for fractured ceramic-fused-to-metal restorations. Journal of Oral Rehabilitation. 2003; 30(2):194-203

17. Ozcan M. Fracture reasons in ceramic-fused-to-metal restorations. Journal of Oral Rehabilitation. 2003;30(3):265-9

18. Salameh Z, Ounsi HF, Aboushelib MN, Sadig W, Ferrari M. Fracture resistance and failure patterns of endodontically treated mandibular molars with and without glass fiber post in combination with a zirconia ceramic crown. Journal of Dentistry. 2008;36(7):513-9

19. Kessler- Liechti G, Mericske-Stern R. Rehabilitation of an abraded occlusion with Procera- $\mathrm{ZrO} 2$ all ceramic crowns. A case report. Schweizer Moatsschrift fur Zahnmedizin. 2006; 116(2):156-67

20. Toksavul S, Turkun M, Toman M. Esthetic enhancement of ceramic crowns with zirconia dowels and cores: a clinical report. The Journal of Prosthetic Dentistry. 2004; 92(2):116-9. 